\title{
Congenital Diaphragmatic Hernia
}

\author{
V.A. Savvina. ${ }^{1,2^{*}}$, A.A. Yashina ${ }^{1}$, A. Yu. Tarasov ${ }^{2}$ \\ ${ }^{1}$ M.K. Ammosov North-Eastern Federal University, Yakutsk, Russia \\ ${ }^{2}$ Republican Hospital №1 - National Center of Medicine, Yakutsk, Russia \\ *Corresponding author. Email: va.savvina@s-vfu.ru
}

\begin{abstract}
Despite significant advances in the treatment of congenital malformations in newborns, there is still a fairly high mortality rate in the group of newborns with congenital diaphragmatic hernia. This fact is associated with the high incidence of concomitant pulmonary hypertension in newborns with lung hypoplasia due to compression by organs that have invaded the chest cavity [1]. In the early 2000s, the mortality rate in congenital diaphragmatic hernia in newborns was approximately $30 \%$. With the introduction of delayed surgery tactics in recent years, survival has improved in the group of infants with hypertension in the pulmonary circulation. The main decisive moments for stabilizing a newborn with congenital diaphragmatic hernia and reducing the degree of pulmonary hypertension are the early transfer of the patient to machine breathing in high-frequency ventilation mode, adherence to the temperature regime, minimization of invasive procedures, and the inclusion of nitric oxide in the breathing mixture as a respiratory vasodilator. The incidence of diaphragmatic hernias varies widely — from 1 in 2000 to 1 in 4000 newborns; this does not take into account a large group of stillborns with diaphragm malformations [2].
\end{abstract}

Keywords: congenital diaphragmatic hernia, congenital malformations, newborns.

\section{INTRODUCTION}

The first description of successful treatment of a newborn with congenital diaphragmatic hernia was demonstrated by Ladd W.E. and Gross R.E. in 1940, when the diaphragm was successfully reconstructed in a child 40 hours after birth. In 1946, Gross R.E. published the first series of diaphragmatic hernia operations, which resulted in 12 of 19 children surviving. For the first time, an endosurgical technique for correcting a diaphragmatic hernia was proposed by David C. van der Zee and Klass $(\mathrm{N})$ M.A. Bax, using a laparoscopic approach with posterolateral localization of the defect. In 2001, a series of surgical corrections of the posterolateral diaphragm defect from the thoracoscopic approach was published by Becmeur F., Jamali R.R., and Moog R. [3].

\section{MATERIALS AND METHODS}

The article analyzes newborns admitted with a diagnosis of congenital diaphragmatic hernia to the Surgical Department of the Pediatric Center at Republican Hospital No. 1 (National Center of
Medicine) from 2010 to 2020. During the study period, 28 newborns were admitted with congenital diaphragmatic hernia. Antenatally, the malformation was detected in $82 \%$ of patients, all cases were confirmed by an expert ultrasound of the fetus at the Medical Genetic Center RH No. 1 (NCM) -23 newborns).

In the 1990s, a technique was proposed for prenatal prediction of cardiopulmonary complications in the presence of a diaphragmatic hernia according to the degree of compression of the contralateral, i.e., healthy lung. The most popular criterion for assessing the postnatal prognosis in diaphragmatic hernia has since become LHR (lungto-head ratio, lung area to head circumference ratio). The right healthy lung with left-sided diaphragmatic hernia is measured by multiplying the maximum diameters, the resulting indicator is correlated with the head circumference measured by scanning in a standard axial plane to assess the biparietal size (clear visualization of M-echo and posterior horns of the lateral ventricles, and the location of the transparent septum cavity at a distance of $1 / 3$ from the frontal bone) [4,5].Among antenatally detected cases, 3 fetuses were diagnosed with a lung-to-head ratio 
(LHR) of $<1.5$, which indicates an extreme degree of hypoplasia of both lungs. At the perinatal council, in these cases, a conversation was held about a possible bad result after the birth of a child, since pulmonary hypertension against the background of extremely pronounced hypoplasia of the lung is the main factor contributing to the lethality of a newborn with congenital diaphragmatic hernia. In two cases, the family decided to prolong the pregnancy. An important component in the formation of postnatal prognosis in congenital diaphragmatic hernia in the fetus is the assessment of the relative position of organs in the chest cavity and especially the position of the liver. When the liver is displaced into the chest cavity, the survival rate is about $40 \%$, with an intact liver the rate is approximately $75 \%$ [6].

Of the 28 newborns, girls accounted for $67 \%$ (19). There were 20 full-term children (82\%), mean gestational age was 37-38 weeks. The average body weight of the newborns was $3,100 \pm 200 \mathrm{~g}$. There were 5 premature children $(18 \%)$, mean gestational age was 33-35 weeks, average body weight was $2500 \pm 200 \mathrm{~g}$.

In these cases, respiratory disorders and cyanosis develop immediately after birth, with acute respiratory failure progressing quickly. On examination, in addition to cyanosis, attention is drawn to the asymmetry of the chest with bulging of the side of the lesion and the absence of excursion in this half. A very characteristic symptom is a sunken scaphoid abdomen. On auscultation, there is either none or sharply weakening breathing. Heart sounds (with left-sided hernia) on the left are heard to the right of the sternum, while with right-sided - along the anterior axillary line on the left, which indicates a displacement of the heart to the healthy side.

Postnatal diagnosis of diaphragmatic hernias is not always straightforward. The greatest importance should be given to X-ray examination. For left-sided phrenic-pleural hernias, ring-shaped clearing is characteristic over the entire left half of the chest, mainly having a spotted pattern. It is difficult to distinguish a false diaphragmatic hernia from a true one, especially if the pleural cavity is filled with intrusive bowel loops. Usually, with true hernias, it is possible to trace the upper contour of the hernial sac radiographically, limiting the eventrated abdominal organs to the chest. If there are difficulties in differential diagnosis, an examination of the gastrointestinal tract with a radiopaque substance (irrigoscopy) is carried out, meanwhile it has to be clear which part of the intestine is in the chest cavity.

Concomitant malformations were identified in 16 cases $(57 \%)$ - mainly congenital heart defects in the form of septal defects, functioning ductus arteriosus, and in 1 case it was a combination of severe coarctation of the aorta, hydronephrotic transformation of the kidneys and sequestration of the lung in 1 child. In almost $90 \%$ of cases, organs were found to move into the cavity of the left hemithorax through the posterior slit-like Bochdaleck defect; in other cases, aplasia of the left dome of the diaphragm was found with displacement into the chest cavity not only of hollow organs, but also of the left lobe of the liver, spleen, and thoracic dystopia of the left kidney ( 3 children). In all cases, a false diaphragmatic hernia without a hernial sac was revealed.

Preoperative examination included physical examination, plain chest x-ray, abdominal echography, neurosonography, and echocardiography measuring the degree of pulmonary hypertension.

\section{RESEARCH RESULTS}

25 newborns with congenital diaphragmatic hernia were operated on, the remaining 3 patients died 1018 hours after birth due to severe respiratory failure and severe pulmonary hypoplasia, with 1 case of cardiopulmonary insufficiency with severe coarctation of the aorta. In the latter case, congenital defect of the diaphragm was detected postnatally. Two children with 1 st day fatality were patients with LHR $<1.5$ and prolonged pregnancy after prenatal consultation. Thus, the antenatal prognostic factor of the LHR indicator was reliable in our cases.

3 patients were diagnosed on echocardiography for 1 st degree of pulmonary hypertension, these patients were planned to be transferred to mechanical ventilation, the duration of preoperative preparation in this group was 1 day. In the presence of signs of pulmonary hypertension of 2-3rd degrees due to severe hypoplasia of the lungs, the newborns were intubated in the delivery room and their preoperative preparation lasted up to 3 days. In recent years, the tactic of the EXIT procedure has been successfully used - transferring the newborn to mechanical ventilation before the first breath, without separating it from the mother's umbilical cord, which allows minimizing hypoxia during resuscitation care.

During preoperative preparation, it is necessary to minimize invasive manipulations with the patient, since excessive production of catecholamines in response to invasive procedures can aggravate vasospasm of the pulmonary circulation and worsen the symptoms of pulmonary hypertension. For the same purpose, it is important on the first day the functioning of fetal vascular communications, in particular the ductus arteriosus, which we prolonged in 1 case with the introduction of prostaglandin E. 
The preoperative preparation algorithm included infusion therapy according to physical needs with the introduction of parenteral nutrition elements from 23 days of life, as well as alkalization elements with the introduction of a $4 \%$ sodium bicarbonate solution with the maintenance of blood $\mathrm{pH}$ above mean indicators to prevent acidosis ( $\mathrm{pH} 7.35$ - 7.45); preparation also included oxygen saturation against the background of high-frequency mechanical ventilation, antibacterial and hemostatic therapy, thermal regime in the conditions of the incubator, maintenance of the light microclimate.

Thus, $88 \%$ of patients ( 22 children) were operated on the 3rd day of life. It is advisable to perform surgery with the following parameters: diuresis $>1$ $\mathrm{ml} / \mathrm{kg} / \mathrm{h}, \mathrm{FiO} 2<0.5$, preductal oxygen saturation 85 $95 \%$, stable mean arterial pressure for gestational age, lactate $<3 \mathrm{mmol} / \mathrm{L}$, calculated pulmonary pressure arteries below the systemic pressure. On the day of the operation, echocardiography was repeated with measurement of the parameters of pulmonary hypertension; in all cases with operated and surviving patients, a decrease in the degree of pulmonary hypertension was achieved during the preoperative preparation. In addition, clinically, on days 2-3 of therapy, stabilization of the patient's condition was also noted, SatO2 was higher than $85 \%$.

The first thoracoscopic plastic surgery of the diaphragm defect in a newborn at the Pediatric Center of the State Autonomous Institution of the Sakha (Yakutia) Republic RH No. 1 - NCM was performed in March 2010. From this period, all newborns with diaphragmatic hernia who were admitted to the clinic were scheduled for thoracoscopic diaphragm surgery [7,8]. Surgical intervention was carried out under standard endotracheal anesthesia in 22 cases, high-frequency oscillatory artificial ventilation of the lungs of newborns in 3 cases, using a KarlStorz endoscopy complex with the patient on their right side. Three patients were operated on with a postero-lateral approach on the left, the reasons for using the traditional approach were prematurity (small volume of hemithorax) in 2 cases and insufficient oxygen saturation in 1 case, since the patient's condition could worsen with the introduction of pneumothorax. The contents of the left pleural cavity in all newborns were loops of the small and large intestine, in 11 cases the spleen and left kidney were also found (Fig. 1).

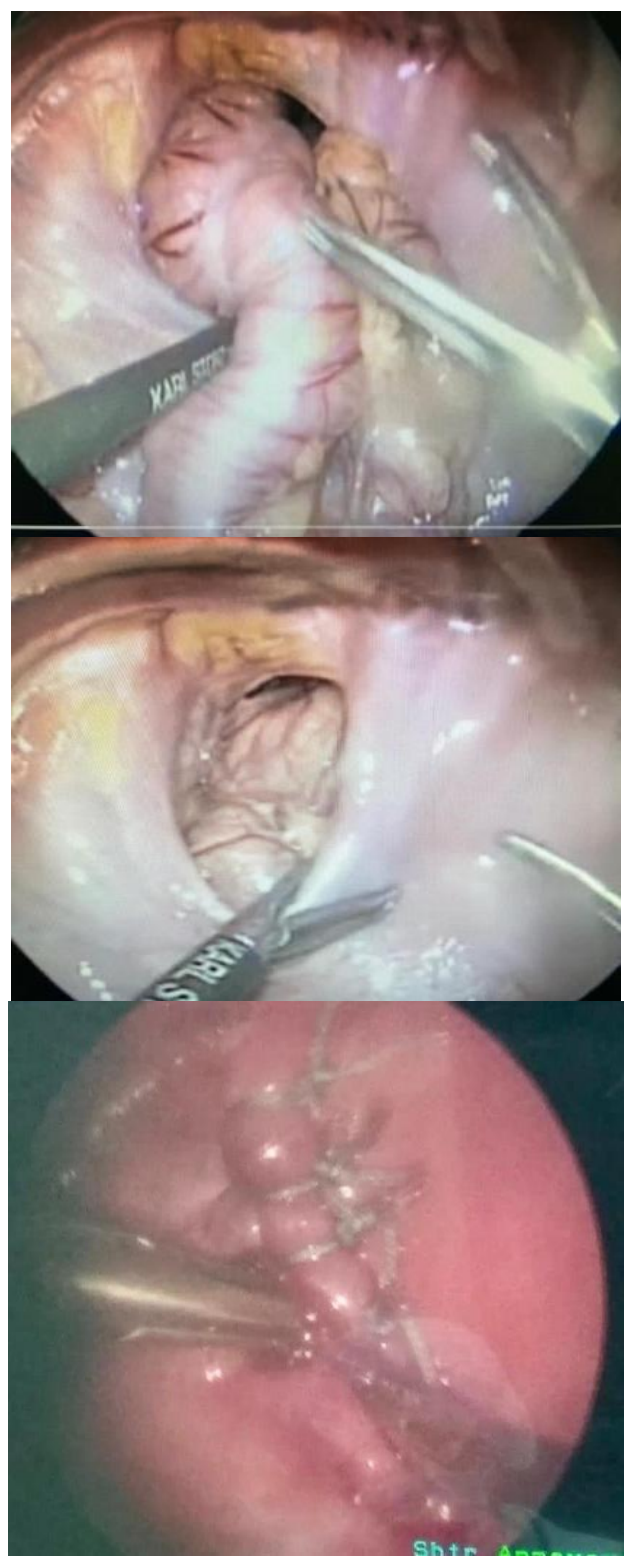

Fig. 1. Stages of thoracoscopic plasty of the left hemithorax defect: A - reduction of intestinal loops into the abdominal cavity, B - posterior diaphragm defect; clear contouring of muscle ridges, $\mathrm{C}$ - diaphragm defect is sutured with interrupted sutures.

The average operation time was $120 \pm 20$ minutes. There were no blood loss or intraoperative complications, and there were no cases of conversion. Thoracoscopy allows you to visualize organs that have moved into the pleural cavity and atraumatically set them into the abdominal cavity. In no case did we encounter technical difficulties in moving organs. Their reduction is facilitated by the insufflation of carbon dioxide into the pleural cavity. The pressure in the pleural cavity at $5 \mathrm{~mm} \mathrm{Hg}$ in the absolute majority of cases allows us to collapse the lung and create optimal conditions for manipulation on the 
diaphragm. Also, it is precisely this pressure that is determined as safe for newborns, it does not cause hemodynamic disturbances. Since unimpeded access to the diaphragm is possible, defect suturing process did not present any technical difficulties. After assessing the diaphragm defect, it is sutured with nonabsorbable Mersilen, Ethibond (Ethicon) $2 / 0$ or $3 / 0$ sutures. We did not notice any fundamental differences between the imposition of interrupted sutures or a continuous suture, as well as the difference in time spent on this procedure. Also, a thoracoscopic approach is preferable due to the lack of dissection of a large array of muscles, which can impair respiratory function in the postoperative period. Thanks to the absence of damage to the peritoneum, there is no postoperative paresis of the gastrointestinal tract and the development of adhesive disease. In 3 cases, plastic surgery of the diaphragm was performed with the synthetic material Permocol due to a deficiency of local tissues in the form of the absence of a muscle cushion (Fig. 2).

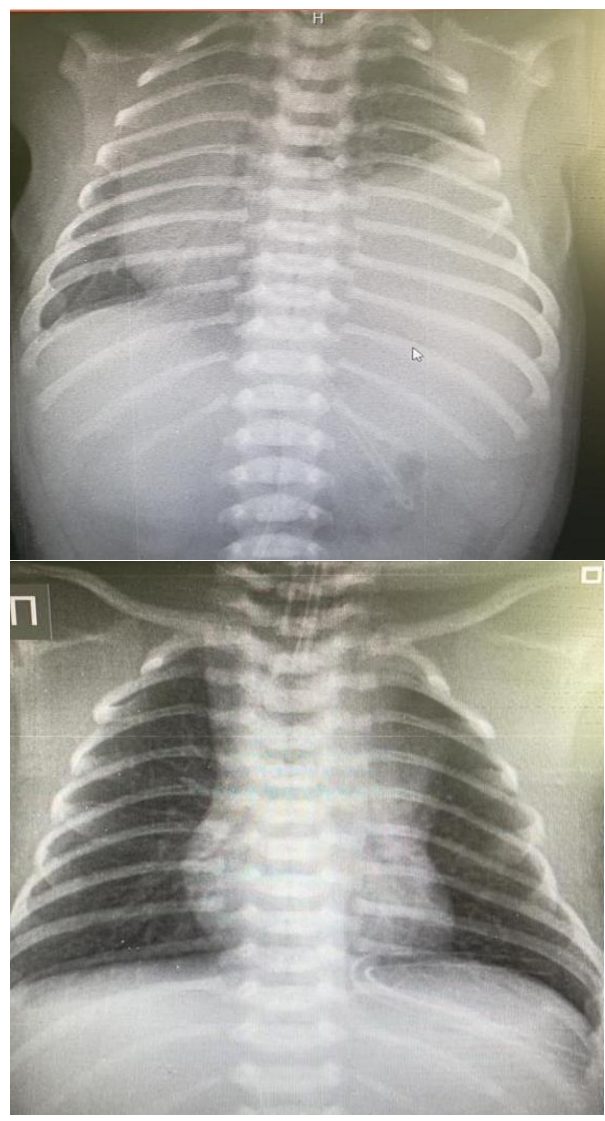

Fig. 2. Chest X-rays: A - before the operation, the left hemithorax occupies the hernial contents, the mediastinum is sharply shifted to the right; $\mathrm{B}-1$ st day after thoracoscopic plasty of the posterior diaphragm defect, the left lung straightened, a small atelectasis of the upper lobe on the left persists, a Bülau drain was installed in the left pleural cavity.

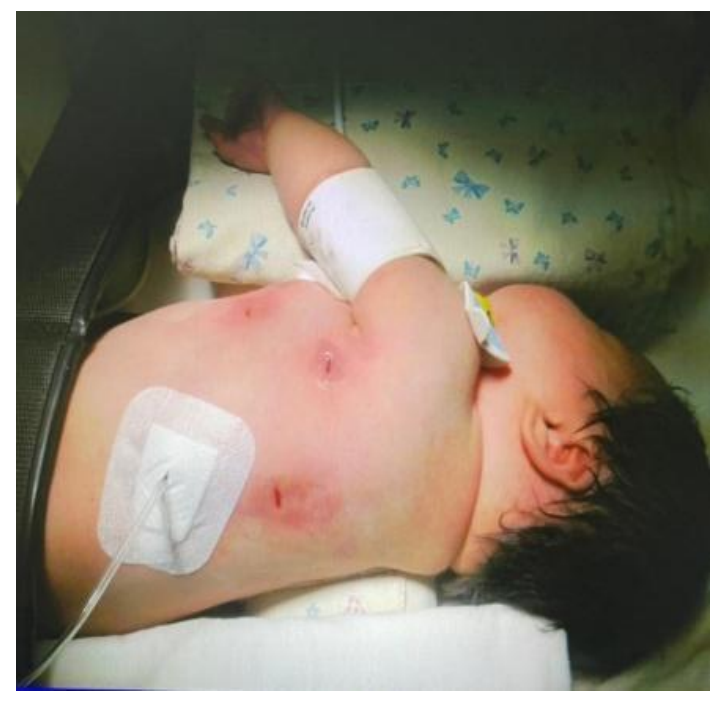

Fig. 3. The patient's appearance after thoracoscopic repair of the diaphragm defect.

Thus, the results of diaphragmatic hernia correction and literature data show that the thoracoscopic method allows performing the primary radical correction of the defect in $90 \%$ of cases [9]. The most common contraindications for this type of surgery are considered to be body weight less than 2.5 $\mathrm{kg}$ and the presence of a stomach or liver in the pleural cavity. The modern endoscopic equipment used makes it possible to obtain a high-definition and detailed image on a large-format monitor. The multiple magnification of the object makes it possible to conduct an audit and monitor the performed manipulations in the pleural cavity, even in newborns with low body weight. The use of carboxytorax with a pressure of 3-6 $\mathrm{mm} \mathrm{Hg}$ with minimum flow values ( $11 / \mathrm{min}$ ) in all cases allows us to continue the intervention using the thoracoscopic method, regardless of the weight of the child. The selection of patients for endoscopic surgery can create an artificial impression about a more favorable course of the postoperative period than with open surgery. The volume of the pleural cavity due to the creation of carboxytorax sufficient not only for imposing interrupted sutures on the edges of the diaphragm defect, but also for repair of the diaphragm dome with synthetic material in case of hypoplasia or aplasia of the diaphragm dome. The anatomical variant of the defect is not a contraindication for endoscopic surgery, even in the case of aplasia of the diaphragm dome and in cases where the use of implant materials is necessary. 
The average duration of artificial lung ventilation after thoracoscopic surgery was 5-6 days, pleural drainage - 4.7 days (Fig. 3). Enteral loading began on days 2-3. The average duration of postoperative hospitalization was $14-15$ bed days. In the early postoperative period, chylothorax developed in two patients and was arrested conservatively. The children were examined in the follow-up: in 2 cases there was a recurrence of hernia at the age of 6 and 7 months, the infants were operated on again with a thoracoscopic approach. The rest of the patients are developing according to their age, the functional state of the diaphragm is satisfactory. All children received good functional and cosmetic results. The mortality rate of newborns with congenital diaphragmatic hernia was $10.7 \%$, all patients who died were with severe pulmonary hypertension which could not be repaired, the lethal outcome occurred on the first day of life.

Until 2000s, in relation to the timing of surgical treatment of newborns with congenital diaphragmatic hernia, the tactic of emergency surgery in the first day of the patient's life was widely used, despite the patient's unstable condition for pulmonary hypertension, since it was believed that only an urgently performed operation could reduce the phenomenon of the so-called asphyxial infringement of the organs in the hemithorax. During that period, the mortality rate in diaphragmatic hernia in newborns reached $30-40 \%$. After a change in the tactics algorithm for newborns with congenital diaphragmatic hernia with a delayed operation to stabilize the patient against the background of ventilation therapy, there is now a 3-fold decrease in mortality for this malformation.

\section{CONCLUSIONS}

Delayed surgery in newborns with congenital diaphragmatic hernia helps to reduce the degree of pulmonary hypertension and improve clinical results.

Sufficient visualization of all stages of the intervention, the absence of technical difficulties in lowering the organs and the actual plastic of the diaphragm, rapid recovery in the immediate postoperative period are the undoubted advantages of endosurgical intervention.

The outcome of such interventions depends on the experience of the doctors involved in the treatment of this extremely difficult category of patients, as well as the development of neonatal anesthesia and resuscitation services in each specific medical institution.

\section{REFERENCES}

[1] P. Bagolan, G. Casaccia, F. Crescenzi et al. J. Pediatr, Surg. 39 (2004) 313-318.

[2] A.Yu. Razumovskiy, O.G. Mokrushina, I.D. Belyayeva et al. Detskaya khirurgiya, 3 (2012) 4-8.

[3] A.Yu. Razumovskiy, O.G. Mokrushina, V.S. Shumikhin et al. Rossiyskiy vestnik detskoy khirurgii, anesteziologii i reanimatologii, 2 (2012) 80-86.

[4] E.V. Yudina, A.Yu. Razumovskiy, O.G. Mokrushina et al. SonoAceUltrasound, 24 (2012) 12-20.

[5] L. Sbragia, B. Paek, R. Filly et al. J. Ultrasound Med., 19 (2000) 845-848.

[6] C.D. Downard, T. Jaksic, J.J. Garza et al. J. Pediatr. Surg., 38 (2003) 729-732.

[7] F. Becmeur, O. Reinberg, C. Dimitriu Semin. Pediatr. Surg., 16(4) (2007) 238-244.

[8] A.Yu. Tarasov, V.A. Savvina, M.E. Okhlopkov et al. Detskaya khirurgiya, 6 (2012) 21-23.

[9] A.Yu. Razumovskiy, O.G. Mokrushina Endokhirurgicheskiye operatsii u novorozhdennykh (MIA, Moscow, 2015) 